\title{
Efecto de la Suplementación con Cobre a Nivel Farmacológico sobre el Comportamiento Productivo, Morfometría Intestinal, Cobre Hepático y Fecal en Cuyes (Cavia porcellus)
}

\author{
Effect of Supplementation with Pharmacological Levels of Copper on \\ Productive Performance, Intestinal Morphometry, and Hepatic and Faecal \\ Copper in Guinea Pigs (Cavia porcellus)
}

Luis Nakandakari A. ${ }^{1}$, Carlos Vílchez P.,3

\section{Resumen}

El estudio tuvo por objetivo evaluar el efecto de niveles del cobre suplementado en niveles farmacológicos sobre el comportamiento productivo, morfometría intestinal, cobre hepático y cobre en heces de cuyes durante 56 días. Se emplearon 60 cuyes machos destetados, distribuidos en cuatro tratamientos con cinco repeticiones por tratamiento y tres animales por repetición: $\mathrm{T} 1$, dieta basal $(0 \mathrm{ppm} \mathrm{Cu}$ suplementado como sulfato de cobre); T2, dieta basal +100 ppm Cu; T3, dieta basal +200 ppm Cu; T4, dieta basal +300 ppm $\mathrm{Cu}$. No se encontró diferencia estadística en peso inicial, peso final, ganancia diaria de peso, consumo voluntario de alimento, conversión alimenticia y mortalidad. La suplementación con cobre causó una reducción significativa $(\mathrm{p}<0.05)$ de la altura de vellosidad intestinal y relación de altura de vellosidad con profundidad de cripta conforme se aumentó el nivel de suplementación. El nivel de cobre hepático y en las heces aumentó significativamente $(\mathrm{p}<0.05)$ conforme aumentaron los niveles de cobre en la dieta. Se concluye que con la suplementación de niveles farmacológicos de cobre en la dieta de cuyes no mejora el comportamiento productivo, incrementa la concentración de cobre tanto en el hígado como en las heces y afecta negativamente la morfometría intestinal.

Palabras clave: cuyes, cobre, morfometría intestinal, histología, hígado, heces

\footnotetext{
${ }^{1}$ Departamento Académico de Veterinaria y Zootecnia, Facultad de Medicina Veterinaria y Zootecnia, Universidad Peruana Cayetano Heredia, Lima, Perú

2 Departamento Académico de Nutrición, Facultad de Zootecnia, Universidad Nacional Agraria La Molina, Lima, Perú

${ }^{3}$ E-mail: cvilchezp@lamolina.edu.pe
}

Recibido: 11 de febrero de 2016

Aceptado para publicación: 16 de mayo de 2016 
The study aimed to evaluate the effect of dietary pharmacological levels of supplementary copper on the productive performance, intestinal morphometry, hepatic copper, and copper in feces of guinea pigs during 56 days. Sixty weaned male guinea pigs were used for this experiment, randomly distributed into four treatments with five replicates each and three animals per replicate: $\mathrm{T} 1$, basal $\operatorname{diet}(0 \mathrm{ppm}$ of $\mathrm{Cu}$ supplemented as copper sulfate); T2, basal diet $+100 \mathrm{ppm} \mathrm{Cu}$; T3, basal diet $+200 \mathrm{ppm} \mathrm{Cu}$; T4, basal diet $+300 \mathrm{ppm}$ $\mathrm{Cu}$. No significant differences were found between treatments on final body weight, daily body weight gain, voluntary feed intake, feed conversion ratio and mortality. In addition, supplemented copper caused a significantly reduction $(\mathrm{p}<0.05)$ of the villus height and villus height:crypt depth ratio as the level of supplemented copper increased. The copper concentration in liver and feces significantly increased $(p<0.05)$ as the level of supplemented copper increased. It is concluded that dietary pharmacological levels of supplementary copper did not improve productive performance, increased the concentration of copper in liver and feces and showed a negative effect on intestinal morphometry.

Key words: guinea pig, copper, intestinal morphometry, histology, liver, feces

\section{INTRODUCCIÓN}

La población nacional y mundial está en constante crecimiento y con ello la necesidad de fuentes alimenticias, tanto de origen animal como vegetal. Esto conlleva la necesidad de mejorar las prácticas productivas con la finalidad de hacer uso más eficiente de los recursos existentes (Cao y Li, 2013). Al respecto, la carne de cuy es una alternativa que está siendo cada vez más importante como fuente de proteína animal (Cawthorn y Hoffman, 2014).

Los antibióticos promotores de crecimiento son aditivos no nutricionales que se vienen empleando por más de 50 años en la producción animal debido a su efecto benéfico sobre la ganancia de peso y conversión alimenticia (Dibner y Richards, 2005); no obstante, en la actualidad se vienen estudiando alternativas al uso de antibióticos debido a que su empleo indiscriminado puede generar resistencia a las enfermedades en la población humana.
El cobre, por otro lado, puede ser usado como promotor de crecimiento ya que es un elemento traza esencial que participa en reacciones metabólicas en el organismo, así como en la formación de hemoglobina para el transporte de oxígeno en la sangre, la producción de energía en la cadena respiratoria mitocondrial, la defensa frente a los radicales libres que dañan a las membranas celulares, y la formación de colágeno y elastina, entre otras funciones (Roberts y Sarkar, 2008).

Se ha demostrado los efectos benéficos del cobre como promotor de crecimiento a niveles farmacológicos sobre las respuestas productivas de aves, cerdos y conejos (European Commission, 2003). Así, por ejemplo, Pérez et al. (2011) reportan que 250 a $315 \mathrm{ppm}$ de cobre tiene un efecto positivo sobre la velocidad de crecimiento en lechones destetados; mientras que en pollos de carne, Zea y Vílchez (2014) mencionan que 250 ppm de cobre tiene un efecto significativo sobre la conversión alimenticia. De igual manera, Adu y Egbunike (2010) concluyen 
que 300 ppm de cobre en la dieta de conejos tuvo efecto positivo como promotor de crecimiento. No obstante, no existe información acerca del suplemento de cobre en el engorde, morfometría intestinal o su acumulación en el hígado de cuyes.

Por lo tanto, el objetivo del presente estudio fue evaluar el comportamiento productivo, morfometría intestinal, cobre hepático y en heces en cuyes de engorde, suplementados con niveles farmacológicos de cobre.

\section{Materiales y Métodos}

El presente estudio se llevó a cabo en la granja de cuyes del Programa de Investigación en Carnes de la Facultad de Zootecnia, Universidad Nacional Agraria La Molina (UNALM), Lima, Perú, durante los meses de abril a junio de 2012, y tuvo una duración de 56 días. Se emplearon 60 cuyes machos destetados (14 a 18 días de edad), distribuidos en cuatro tratamientos con cinco repeticiones y tres animales por repetición. Los animales estuvieron alojados en 20 pozas con un área de $0.1 \mathrm{~m}^{2}$ por cuy, construidas artesanalmente a base de ladrillo en piso de concreto.

Los tratamientos fueron: $\mathrm{T} 1$, dieta basal (0 ppm Cu suplementado como sulfato de cobre); T2, dieta basal +100 ppm Cu; T3, dieta basal $+200 \mathrm{ppm} \mathrm{Cu}$; T4, dieta basal + $300 \mathrm{ppm} \mathrm{Cu}$. La dieta basal fue el alimento integral comercial «La Molina» para cuyes, de la Planta de Alimentos del Programa de Investigación en Alimentos de la Facultad de Zootecnia de la UNALM, cuyo valor nutricional calculado se presenta en el Cuadro 1. La presentación de la dieta basal fue a base de harina, teniendo como ingredientes maíz amarillo, torta de soya 48 , pasta de algodón, torta de girasol, subproductos de trigo, heno de alfalfa, carbonato de calcio, fosfato dicálcico, aminoácidos sintéticos, vitaminas, minerales y sal. Para la preparación
Cuadro 1. Valor nutritivo calculado de dieta basal empleada en la etapa de crecimiento - engorde en cuyes

\begin{tabular}{lc}
\hline Valor nutricional (calculado) & $\begin{array}{c}\text { Dieta } \\
\text { basal }\end{array}$ \\
\hline E. digestible, Mcal/kg, Mín & 2.80 \\
Proteína bruta, \%, Mín & 18.00 \\
Fibra cruda, \%, Mín & 8.00 \\
Calcio, \%, Máx & 0.80 \\
Fósforo total, \%, Mín & 0.80 \\
Sodio, \%, Mín & 0.20 \\
Lisina, \%, Mín & 0.84 \\
Metionina - Cistina, \%, Mín & 0.60 \\
Arginina, \%, Mín & 1.20 \\
Treonina, \%, Mín & 0.60 \\
Triptófano, \%, Mín & 0.18 \\
Ac. ascórbico, mg/100 & 20.00 \\
Cobre, ppm & 10.00 \\
\hline
\end{tabular}

de las dietas experimentales de los tratamientos 2, 3 y 4 se realizó el cálculo de la cantidad requerida para suplementar 100, 200 y 300 ppm de cobre en la dieta.

El contenido de cobre en el alimento se determinó por espectrofotometría de absorción atómica siguiendo la metodología de la Normal Oficial Mexicana (NOM-117-SSA11994), el cual se realizó en el Laboratorio de Suelos, Plantas, Aguas y Fertilizantes (LASPAF) de la Facultad de Agronomía de la UNALM. La determinación del contenido de cobre en el hígado y en las heces fue realizada por medio de un espectrofotómetro de absorción atómica siguiendo una metodología similar.

El alimento fue ofrecido en forma diaria. Además, se proporcionó agua limpia y fresca ad libitum. La cantidad de alimento ofrecido y sobrante del día anterior fue registrada para calcular el consumo de alimento. Asimismo, se registró el peso vivo una vez 
por semana y en ayunas (07:00) y se calculó la ganancia diaria de peso. Para el registro del peso del alimento y de los cuyes se empleó una balanza digital con capacidad máxima de $5 \mathrm{~kg}$ y una precisión de $0.05 \mathrm{~g}$.

Culminado los 56 días de crianza fueron sacrificados dos cuyes por repetición para realizar mediciones citomorfométricas e histológicas. Para este fin, se colectó un segmento del intestino delgado $(2 \mathrm{~cm})$, ubicado en el punto medio del yeyuno, tomando como referencia la parte inicial del yeyuno a nivel de la desembocadura del conducto biliar y la parte final del yeyuno en la unión yeyunoíleon, a nivel del divertículo de Meckel. La muestra se almacenó con formol al 10\% y fue trasladada al Laboratorio de Histología y Patología de la Facultad de Veterinaria y Zootecnia, Universidad Peruana Cayetano Heredia (FAVEZ-UPCH) para su procesamiento histológico. Las mediciones se hicieron siguiendo el protocolo de evaluación indicado por Bernal (2011), tomándose el promedio de 10 vellosidades. Las mediciones de altura de vellosidad y profundidad de cripta se hicieron a un aumento de 10x. Con los resultados se calculó la relación altura de vellosidad con profundidad de cripta.

Para la evaluación histológica del tejido hepático se extrajeron muestras de $2 \mathrm{~cm}^{2}$ del hígado, las que fueron llevadas al Laboratorio de Histología y Patología de la FAVEZ$\mathrm{UPCH}$. Las muestras fueron procesadas mediante métodos estándares y se prepararon las láminas histológicas. Usando un microscopio de campo claro, se evaluaron los cambios histológicos a nivel de la arquitectura del tejido hepático, empleando aumentos de 4, 10 y 40x. Los cambios del patrón celular del parénquima hepático fueron asignados en una escala de 0 a 4 (Bernal, 2011), siendo 0 : no presencia de daño tisular; 1 : presencia de degeneración tisular más congestión sanguínea; 2: presencia de necrosis focal; 3: presencia de necrosis multifocal; 4: presencia de tejido fibroso.
Se empleó un diseño completamente al azar con cuatro tratamientos y cinco repeticiones por tratamiento. El análisis de varianza se llevó a cabo usando el paquete estadístico Statistical Analysis System (SAS, 1999) y la comparación de medias se realizó utilizando la prueba de Duncan. Para la evaluación histológica del tejido hepático de características no paramétricas, se realizó el análisis de regresión logística utilizando el programa $\mathrm{R}$ Project for Statistical Computing 2.6.1 (R Development Core Team, 2006).

\section{Resultados y Discusión}

No se encontró diferencia estadística entre los grupos en los parámetros de producción bajo evaluación (Cuadro 2), lo cual coincide con otros estudios en pollos de carne (Almeida et al., 2003) y lechones (Hedemann et al., 2006). En dichos estudios, los autores sostienen que los resultados se deben al buen estado sanitario de las instalaciones y a la buena salud de los animales, como ocurrió en este experimento. No obstante, Espíritu (1990) encontró un efecto positivo $(\mathrm{p}<0.05)$ sobre la ganancia de peso, consumo de alimento y conversión alimenticia alimentando cuyes con cobre entre $100 \mathrm{y}$ $400 \mathrm{ppm}$, con la diferencia de haber usado una dieta mixta de forraje-concentrado y un tiempo experimental de 84 días.

La suplementación con cobre tuvo un efecto negativo en la altura de vellosidad, profundidad de cripta y relación de altura de vellosidad con profundidad de cripta en T2, T3 y T4 sobre T1 $(\mathrm{p}<0.05)$ (Cuadro 3$)$, donde los valores decrecen con el incremento de $\mathrm{Cu}$ suplementado.

Estos resultados coinciden con Fry et al. (2012), quienes encontraron una disminución en la altura de vellosidad a nivel del duodeno y yeyuno proximal a una suplementación de $225 \mathrm{ppm}$ de cobre en la dieta de cerdos destetados. Este autor señala que el cobre 
Cuadro 2. Efecto del cobre (ppm de Cu suplementado) sobre el comportamiento productivo de cuyes ${ }^{1}$ en periodo de 56 días de engorde

\begin{tabular}{lcccc}
\hline & $0 \mathrm{ppm}$ & $100 \mathrm{pm}$ & $200 \mathrm{ppm}$ & $300 \mathrm{ppm}$ \\
& $(\mathrm{T} 1)$ & $(\mathrm{T} 2)$ & $(\mathrm{T} 3)$ & $(\mathrm{T} 4)$ \\
\hline Peso inicial $(\mathrm{g})$ & $289^{2 \mathrm{a}}$ & $268 \mathrm{a}^{\mathrm{a}}$ & $307 \mathrm{a}^{\mathrm{a}}$ & $296^{\mathrm{a}}$ \\
Peso final (g) & $903^{\mathrm{a}}$ & $868^{\mathrm{a}}$ & $911^{\mathrm{a}}$ & $920^{\mathrm{a}}$ \\
Ganancia de peso (g/día) & $10.9^{\mathrm{a}}$ & $10.7^{\mathrm{a}}$ & $10.8^{\mathrm{a}}$ & $11.2^{\mathrm{a}}$ \\
Consumo de alimento (g/día) & $48.5^{\mathrm{a}}$ & $51.6^{\mathrm{a}}$ & $54.5^{\mathrm{a}}$ & $55.3^{\mathrm{a}}$ \\
Conversión alimenticia & $4.42^{\mathrm{a}}$ & $4.82^{\mathrm{a}}$ & $5.07^{\mathrm{a}}$ & $4.96^{\mathrm{a}}$ \\
\hline
\end{tabular}

${ }^{1}$ Valores son promedio de cinco repeticiones, un animal por repetición

${ }^{a}$ Superíndices similares dentro de filas indican ausencia de diferencia estadística

Cuadro 3. Efecto del suplemento de cobre ${ }^{1}$ sobre altura de vellosidad, profundidad de cripta y relación de altura con profundidad a nivel de yeyuno medio en el cuy

\begin{tabular}{lccc}
\hline & $\begin{array}{c}\text { Altura de } \\
\text { vellosidad }(\mu \mathrm{m})\end{array}$ & $\begin{array}{c}\text { Profundidad de } \\
\text { cripta }(\mu \mathrm{m})\end{array}$ & $\begin{array}{c}\text { Relación de } \\
\text { altura con } \\
\text { profundidad }\end{array}$ \\
\hline T1 - Dieta con 0 ppm & $999.4^{2 \mathrm{a}}$ & $235.4^{\mathrm{a}}$ & $4.25^{\mathrm{a}}$ \\
$\mathrm{T} 2$ - Dieta con 100 ppm & $835.2^{\mathrm{b}}$ & $214.0^{\mathrm{b}}$ & $3.90^{\mathrm{b}}$ \\
T3 - Dieta con 200 ppm & $606.2^{\mathrm{c}}$ & $168.2^{\mathrm{c}}$ & $3.61^{\mathrm{c}}$ \\
$\mathrm{T} 4$ - Dieta con 300 ppm & $422.6^{\mathrm{d}}$ & $172.6^{\mathrm{c}}$ & $2.46^{\mathrm{d}}$ \\
\hline
\end{tabular}

${ }^{1}$ Valores son promedio de cinco repeticiones, un animal por repetición

${ }^{a, b}$ Superíndices diferentes dentro de columnas indican diferencia estadística $(p<0.05)$

puede actuar como agente prooxidante y estar involucrado en la oxidación de los lípidos y el estrés oxidativo sobre las membranas celulares. En su estudio se midió concentración de malondialdehído (MDA), el cual es un indicador que se emplea para la determinación del grado de oxidación de los lípidos a causa de un agente oxidante como, por ejemplo, los radicales libres. Esto estaría ocasionando un daño en la membrana celular causando la muerte de los enterocitos y, por ende, una reducción de la altura de vellosidad. De igual manera, Hodgkinson y Petris (2012) señalan que el cobre tiene la capacidad de aceptar y donar electrones en presencia de oxígeno, formando radicales hidroxilos por medio de la reacción de Haber y Weiss, causando daño a lípidos, proteínas y ácidos nucleicos.

Por otro lado, Zhao et al. (2007) y Zea y Vílchez (2014) encontraron un efecto benéfico del cobre sobre la morfometría intestinal en lechones destetados y en pollos, respectivamente. La diferencia entre esos estudios y el presente podría deberse al mejor estado sanitario en que se desarrollaron los experimentos, donde no hubo cuadros de diarrea y ausencia de alimento no digerido en las heces. 


\section{Conclusiones}

- El cobre en la dieta a niveles farmacológicos $(100,200,300 \mathrm{ppm})$ no mostraron influencia significativa sobre el comportamiento productivo de los cuyes.

- La suplementación de cobre en la dieta tuvo efecto negativo sobre la altura de vellosidades intestinales, profundidad de cripta y la relación altura con profundidad.

- La suplementación de cobre a niveles farmacológicos en la dieta resultó en una mayor retención de cobre a nivel hepático y excreción en heces.

\section{Literatura Citada}

1. Adu O, Egbunike G. 2010. Enhancing growing rabbits performance with diets supplemented with cooper. Advan Biol Res 4: 18-22.

2. Almeida M, Machado J, Martínez M, Daroz S. 2003. Cupric citrate as growth promoter for broiler chickens in different rearing stages. Sci Agric 60: 441-445. doi: 10.1590/S0103-90162003000300004

3. Bernal S. 2011. Evaluación del aceite esencial de orégano (Origanum vulgare) y jengibre deshidratado (Zingiber officinale) como alternativa al uso de antibióticos promotores de crecimiento en pollos de engorde. Tesis de Médico Veterinario y Zootecnista. Lima: Univ Peruana Cayetano Heredia. 40 p.

4. Bowland J, Braude R, Chamberlain A, Glascock R, Mitchell K. 1961. The absorption, distribution and excretion of labelled copper in young pigs given different quantities, as sulphate or sulphide, orally or intravenously. Brit J Nutr 15: 59-72.

5. Cao Y, Li D. 2013. Impact of increased demand for animal protein products in Asian countries: implications on global food security. Animal Frontiers 3: 48-55. doi: 10.2527/af.2013-0024.
6. Castell A, Allen R, Beames R, Bell J, Belzile R, Bowland J, Elliot J, Ihnat $M$, Larmond E, Mallard T, Spurr D, Stothers S, Wilton S, Young L. 1975. Copper suplementation of Canadian diets for growing - finishing pigs. J Anim Sci 55:113-134.

7. Cawthorn D, Hoffman L. 2014. The role of traditional and non-traditional meat animals in feeding a growing and evolving world. Anim Frontiers 4: 6-12. doi: 10.2527/af.2014.0027

8. Dibner J, Richard J. 2005. Antibiotic growth promoters in agricultura: history and mode of action. Poult Sci 84: 634643. doi: $10.1093 / \mathrm{ps} / 84.4 .634$

9. Espíritu G 1990. Uso del sulfato de cobre como promotor de crecimiento en cuyes destetados. Tesis de Ing. Zootecnista. Huancayo. Perú: Univ Nac del Centro del Perú. 47 p.

10. European Commission. 2003. Opinion of the Scientific Committee for Animal Nutrition on the use of copper in feeding stuffs. [Internet]. Available in: http:// www.ec.europa.eu/food/fs/sc/scan/ out115_en.pdf

11. Fry R, Ashwell M, Lloyd K, OÈNan T, Flowers W, Stewart K, Spears J. 2012. Amount and source of dietary copper affects small intestine morphology, duodenal lipid peroxidation, hepatic oxidative stress, and mRNA expression of hepatic copper regulatory proteins in weanling pigs. J Anim Sci 90: 3112-3119. doi: $10.2527 /$ jas.2011-4403

12. Hedemann M, Jensen B, Poulsen H. 2006. Influence of dietary zinc and copper on digestive enzyme activity and intestinal morphology in weaned pigs. $J$ Anim Sci 84: 3310-3320.

13. Hodgkinson V, Petris M. 2012. Copper homeostasis at the host-pathogen interface. J Biol Chem 287: 13549-13555. doi: 10.1074/jbc.R111.316406

14. Iqbal R, Malik F, Aziz T, Sarfraz I, Ahmed Z, Shafqat S. 2012. The study of histopathological changes upon exposure to vinegerized copper sulphate in liver and kidney of broiler chick. 
Middle East J Sci Res 12: 36-41. doi: 10.5829/idosi.mejsr.2012.12.1.1648

15. Luo X, Lin J, Steward Y, Lu F, Liu L, Yu S. 2005. Effects of dietary supplementation with copper sulfate or tribasic copper chloride on broiler performance, relative copper bioavailabiliy, and ovidation stability of vitamin $E$ in feed. Poult Sci 84: 888-893.

16. Norma Oficial Mexicana NOM-117SSA1-1994. Bienes y servicios. Método de prueba para la determinación de cadmio, arsénico, plomo, estaño, cobre, zinc y mercurio en alimentos, agua potable y agua purificada por espectofotometría de absorción atómica. [Internet]. Disponible en: http://www.salud.gob.mx/ unidades $/ \mathrm{cdi} / \mathrm{nom} / 117 \mathrm{ssa} 14 . \mathrm{html}$

17. Pérez V, Waguespack A, Bidner T, Southern L, Fakler T, Ward T, Steidinger M, Pettigrew J. 2011. Additivity of effects from dietary copper and zinc on growth performance and fecal microbiota of pigs after weaning. J Anim Sci 89: 414-425. doi: 10.2527/ jas.2010-2839
18. Roberts E, Sarkar B. 2008. Liver as a key organ in the supply, storage, and excretion of copper. Am J Clin Nutr 88: $851 \mathrm{~S}-854 \mathrm{~S}$.

19. $R$ Development Core Team. 2006. R: A language and environment for statistical computing. [Internet]. Available in: http://www.R-project.org

20.SAS Institute.1990. SAS@ User's guide statistics. v. 6.04. Cary, NC, USA: SAS Institute.

21. Zea O, Vílchez C. 2014. Efecto de la suplementación con fuentes de cobre sobre el comportamiento productivo, morfometría intestinal y nivel de cobre hepático en pollos de carne. Rev Inv Vet Perú 25: 16-28. doi: 10.15381/ rivep.v25i1.8464

22. Zhao J, Harper A, Estienne M, Webb K, McElroy A, Denbow D. 2007. Growth performance and intestinal morphology responses in early weaned pigs to supplementation of antibiotic-free diets with an organic copper complex and spray-dried plasma protein in sanitary and nonsanitary environments. J Anim Sci 85: 1302-1310. 